\title{
Hyers-Ulam-Rassias-Wright Stability for Fractional Oscillation Equation
}

\author{
Zahra Eidinejad 1 and Reza Saadati \\ School of Mathematics, Iran University of Science and Technology, Narmak, Tehran 13114-16846, Iran \\ Correspondence should be addressed to Reza Saadati; rsaadati@eml.cc
}

Received 17 November 2021; Accepted 13 December 2021; Published 8 February 2022

Academic Editor: Rodica Luca

Copyright (c) 2022 Zahra Eidinejad and Reza Saadati. This is an open access article distributed under the Creative Commons Attribution License, which permits unrestricted use, distribution, and reproduction in any medium, provided the original work is properly cited.

In this paper, we consider the nonhomogeneous fractional delay oscillation equation with order $\sigma$ and introduce a class of control functions, i.e., Wright functions. Next, we apply the Cădariu-Radu method to prove the existence of a unique solution and HyersUlam-Rassias-Wright stability of the fractional delay oscillation equation. At the end of the article, by an example, we show the application of the obtained results.

\section{Introduction}

From the past until now, fractional calculus has gained considerable popularity and importance due to its many applications in various and wide scientific and engineering fields (see $[1,2]$ ). We consider the following nonhomogeneous fractional delay oscillation equation:

$$
\begin{gathered}
D_{0}^{\sigma} m(\varrho)=-\vartheta m(\varrho-\mu)+g(\varrho)+\mathfrak{£}^{-1}(m(s))(\varrho), \quad \varrho \in \mathfrak{B}:=[0, B], \\
m(\varrho)=\psi(\varrho), m^{\prime}(\varrho)=\psi^{\prime}(\varrho), \quad-\mu \leq \varrho \leq 0,
\end{gathered}
$$

where

(1) $g: \mathfrak{B} \times \mathbb{R}^{n} \longrightarrow \mathbb{R}^{n}$ is an integrable function, $m(\varrho) \in \mathbb{R}^{n}, \vartheta \in \mathbb{R}^{n \times n}$, and $\psi \in C^{2}\left([-\mu, 0], \mathbb{R}^{n}\right)$ denotes constant matrix; also, $\mu>0$ is a fixed time and $B=N \mu$ for a fixed $N \in\{1,2, \ldots\}$.

(2) $D_{0}^{\sigma}$ is the Caputo fractional derivative with $f \in(1,2)$ defined by

$$
\left.D_{0}^{\sigma} m(\varrho)=\int_{0}^{\varrho} \frac{(\varrho-\lrcorner)^{1-\sigma}}{\Gamma(2-\sigma)} m^{\prime \prime}(\beth) \mathrm{d}\right\lrcorner,
$$

if the integral exists.
(3) The classical Laplace transform is defined by the integral formula

$$
(\mathfrak{g})(s)=\int_{0}^{\infty} g(\varrho) e^{-s \varrho} d \varrho
$$

where $g(\varrho)$ is absolutely integrable on $[0, \infty)$. Let $J_{0}^{\sigma}$ denote the Riemann-Liouville fractional integral operator of order $\sigma \in(1,2)$. Assume the Laplace transforms of $J_{0}^{\sigma} g(\varrho), D_{0}^{\sigma} g(\varrho)$ exist for $\varrho \leq 0$ and the Laplace transform of $g(\varrho-b)$ exists for $\varrho \leq b$. Then, we have

(i) $-\mathfrak{E}\left(J_{0}^{\sigma} g(\varrho)\right)(s)=s^{-\sigma} \mathfrak{E}(g(\varrho))(s)$,

(ii) $\mathfrak{E}\left(g_{1}(\varrho)\right)(s)=e^{-\mathrm{bs}} \int_{-\mathrm{b}}^{0} e^{-\varrho s}(\varrho) d \varrho+e^{-\mathrm{b} s} \mathfrak{E}(g(\varrho))$ $(s)$,

(iii) $-\mathfrak{E}\left(g_{1}(\varrho)\right)(s)=e^{-\mathrm{b} s} \int_{-\mathrm{b}}^{0} e^{-\varrho s} g(\varrho) d \varrho+e^{-\mathrm{b} s} \mathfrak{E}(g(\varrho))$ $(s)$,

where $\mathfrak{E}^{-1}$ is the Laplace inverse transform.

Exact solutions for the differential equation using the exponential function for $\sigma=1$ and the matrix sine and matrix cosine for $\sigma=2$ are obtained in $[3,4]$. In 2018, $\mathrm{Li}$ and Wang obtained the exact solution of equation (1) for $0<\sigma<1$ using the Mittag-Leffler type matrix function [5-7]. In the second section of the paper, we present the required 
definitions and concepts. In the third section, we use the alternative fixed point method and apply the Wright function as a control function to investigate the HyersUlam-Rassias-Wright stability for equation (1). Finally, by an example, we show the application of the obtained results.

\section{Preliminaries}

In this section, we present several facts that we need to get the desired results. Since the control function used in this article is a Wright function, we start this section with a definition of the Wright function.

Definition 1. The Wright function is defined by the following series representation:

$$
Z W_{\sigma, \kappa}(q)=\sum_{\ell=0}^{\infty} \frac{q^{\ell}}{\ell ! \Gamma(\sigma \ell+\kappa)},
$$

for $\sigma>-1, \kappa>0, q \in \mathbb{R}$. It is an entire function of order $1 /(1+\sigma)$, which has been known also as generalized Bessel (or Bessel Maitland) function [8,9].
Since we use two functions called the sine-type Mittag-Leffler and the cosine-type Mittag-Leffler and these two functions are derived from the extension of Mittag-Leffler function, we first provide a definition of the Mittag-Leffler function, then we state the main definitions.

Definition 2. The special function

$$
E_{\sigma}(k)=\sum_{\ell=0}^{\infty} \frac{k^{\ell}}{\Gamma(\sigma \ell+1)}, \sigma>0, k \in \mathbb{R}^{n \times n},
$$

and its general form

$$
E_{\sigma, \kappa}(k)=\sum_{\ell=0}^{\infty} \frac{k^{\ell}}{\Gamma(\sigma \ell+\kappa)}, \sigma, \kappa>0, k \in \mathbb{R}^{n \times n} .
$$

Definition 3. The cosine-type Mittag-Leffler function of a polynomial of degree $\mathfrak{p} \sigma, C_{\sigma}^{\mu}(\varrho): \mathbb{R} \longrightarrow \mathbb{R}^{n}$, for $n \in \mathbb{N}$ and $\sigma \in \mathbb{R}^{+}$on the interval $[(\mathfrak{p}-1) \mu, \mathfrak{p} \mu)$ and for $\varrho=\mathfrak{p} \mu, \mathfrak{p}=$ $0,1,2, \ldots, N$ defined by

$$
C_{\sigma}^{\mu}(\varrho):=\left\{\begin{array}{ll}
\Theta, & -\infty<\varrho<\mu, \\
I, & -\mu<\varrho<0, \\
\cdots & \\
I-\vartheta \frac{\varrho^{\sigma}}{\Gamma(\sigma+1)}+\vartheta^{2} \frac{(\varrho-\mu)^{2 \sigma}}{\Gamma(2 \sigma+1)}+\cdots & +(-1)^{\mathfrak{p}} \vartheta^{\mathfrak{p}} \frac{(\varrho-(\mathfrak{p}-1) \varrho)^{\mathfrak{p} \sigma}}{\Gamma(\mathfrak{p} \sigma+1)}, \quad(\mathfrak{p}-1) \mu \leq \varrho<k \mu,
\end{array} .\right.
$$

that $I$ denotes a unit matrix and $\Theta$ denotes a zero matrix.

Definition 4. The sine-type Mittag-Leffler function of a polynomial of degree $\mathfrak{p} \sigma+1, S_{\sigma}^{\mu}(\mathrm{Q}): \mathbb{R} \longrightarrow \mathbb{R}^{n}$, for $n \in \mathbb{N}$ and $\sigma \in \mathbb{R}^{+}$on the interval $[(\mathfrak{p}-1) \mu, \mathfrak{p} \mu)$ and for $\varrho=\mathfrak{p} \mu, \mathfrak{p}=$ $0,1,2, \ldots, N$ defined by

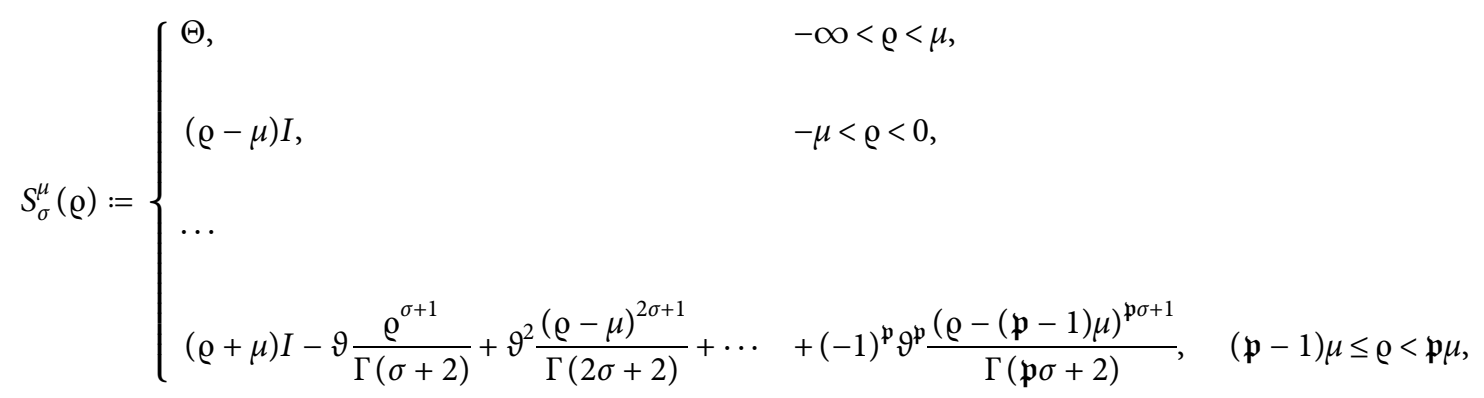


that $I$ denotes a unit matrix and $\Theta$ denotes a zero matrix.

For the homogeneous fractional oscillation system,

$$
D_{0}^{\sigma} m(\varrho)=-9 m(\varrho-\mu), \varrho \in \mathfrak{B} .
$$

We present the following Lemma from [10].

Lemma 1. For $C_{\sigma}^{\mu}(\varrho)$ and $S_{\sigma}^{\mu}(\varrho)$, we find

(i) $d / d \varrho S_{\sigma}^{\mu}(\varrho)=C_{\sigma}^{\mu}(\varrho)$ for all $\varrho \in \mathbb{R}$.

(ii) $C_{\sigma}^{\mu}(\varrho)$ is a solution of (1), $\varrho \in \mathfrak{B}$.

(iii) $S_{\sigma}^{\mu}(\varrho)$ is a solution of (1), $\varrho \in \mathfrak{B}$.
Theorem 5. If the function $m$ is a solution of equation (1) with initial conditions (2), then the function $m$ is defined as follows:

$$
m(\varrho)=\left(s^{\sigma} I+\vartheta e^{-s \mu}\right) \mathfrak{E}(m(\varrho))(s)-s^{\sigma-1} \psi(0),
$$

$$
-s^{\sigma-2} \psi^{\prime}(0)+\vartheta e^{-s \mu} \int_{-\mu}^{0} e^{-s \mu} \psi(\varrho) \mathrm{d} \varrho-\mathfrak{E}(g(\varrho))(s)
$$

Proof. Take the Laplace transform to both sides of (1) and according to Laplace's definitions, we get

$$
s^{\sigma} \mathscr{L}(m(\varrho))(s)-s^{\sigma-1} m(0)-s^{\sigma-2} m^{\prime}(0)=-9 e^{-s \mu}\left(\int_{-\mu}^{0} e^{-s z} y(z) d z+\mathscr{L}(m(\varrho))(s)\right)+\mathscr{L}(g(\varrho))(s)+m(\varrho) .
$$

We solve the above equation for $\mathscr{L}(m(\varrho))(s)$; we get

$$
\left(s^{\sigma} I+\vartheta e^{-s \mu}\right) \mathscr{L}(m(\varrho))(s)=s^{\sigma-1} \psi(0)+s^{\sigma-2} \psi^{\prime}(0)-\vartheta e^{-s \mu} \int_{-\mu}^{0} e^{-s z} \psi(z) \mathrm{d} z+\mathscr{L}(g(\varrho))(s)+m(\varrho),
$$

then

$$
m(\varrho)=\left(s^{\sigma} I+\vartheta e^{-s \mu}\right) \mathscr{L}(m(\varrho))(s)-s^{\sigma-1} \psi(0)-s^{\sigma-2} \psi^{\prime}(0)+\vartheta e^{-s \mu} \int_{-\mu}^{0} e^{-s z} \psi(z) \mathrm{d} z-\mathscr{L}(g(\varrho))(s) .
$$

The following alternative fixed point theorem was proved by Diaz-Margolis [11]; for more results on fixed point theory and application, we refer to [12-14].

Theorem 6. Let $(\mathfrak{S}$ be a set with a complete $[0, \infty]$ valued metric $d$. For $m, \bar{m} \in \mathfrak{G} Z m, \bar{m} \in \mathfrak{G}$, consider the self-map $\widetilde{\sigma}$ on $\mathbb{G}$ such that the following relation holds:

$$
d(\widetilde{\sigma}, \widetilde{\sigma m}) \leq \gamma d(\bar{m}, m)
$$

where $\mathrm{Q}<1$ is a Lipschitz constant. Let $m \in \mathfrak{G}$. Therefore, one of the following two conditions is established:

(i) $d\left(\widetilde{J}^{e} m, \sigma^{e+1} m\right)=\infty, \quad \forall e \in \mathbb{N}$, or

(ii) We can find $\mathbf{e}_{0} \in \mathbb{N}$ such that $d\left(\sigma^{e} m, \sigma^{e+1} m\right)$ $<\infty, \quad \forall e \geq e_{0}$,

which implies that

(1) The fixed point $\bar{m}^{*}$ of $\sigma$ is the convergence point of the sequence $\left\{\widetilde{\sigma}^{e} m\right\}$;

(2) In the set, $\mathfrak{V}^{*}=\left\{\bar{m} \in \mathfrak{G} \mid d\left(\widetilde{J}^{e_{0}} m, \bar{m}\right)<\infty\right\}, \bar{m}^{*}$ is the unique fixed point of $\sigma$;
(3) $(1-\gamma) d\left(\bar{m}, \bar{m}^{*}\right) \leq d(\bar{m}, \sigma \bar{m})$ for every $\bar{m} \in \mathfrak{W}$.

In this article, we use the norm $\|s\|=\sum_{i=1}^{\ell}\left|s_{i}\right|, s \in \mathbb{R}^{n \times 1}$ and the matrix norm.

In the sequel, we provide a definition of Hyers-UlamRassais-Wright stability; for more details and result, we refer to [15-18].

Definition 7. We say that equation (1) is Hyers-UlamRassais-Wright stable on $\mathfrak{B}$ with respect to $W_{\sigma, \kappa}(\varrho)$, if there exists $\mathfrak{E}>0$ such that for each $\beth>0$ and for each solution $\bar{m}(\varrho)$ satisfying inequality

$\left\|D_{0}^{\sigma} \overline{\bar{m}}(\varrho)+9 \bar{m}(\varrho-\mu)-g(\varrho)-\mathfrak{E}^{-1}(\bar{m}(s))(\varrho)\right\| \leq \beth W_{\sigma, \kappa}(\varrho), \quad \varrho \in \mathfrak{B}$,

there exists a solution $m(\varrho)$ of the problem (1) such that

$$
\|\bar{m}(\varrho)-m(\varrho)\| \leq \mathscr{G} \beth W_{\sigma, \kappa}(\varrho),
$$

for every $\varrho \in \mathfrak{B}$.

Next, using the Diaz-Margolis theorem we prove the Hyers-Ulam-Rassais-Wright stability for equation (1). 


\section{Hyers-Ulam-Rassias-Wright Stability of the Fractional Oscillation Equation}

In this section, we investigate the existence of a unique solution and Hyers-Ulam-Rassais-Wright stability for equation (1).

Theorem 8. Consider the positive constant $x$, such that $0<\boldsymbol{x}<1$. Consider the control function $W_{\sigma, \kappa}: \mathfrak{B} \longrightarrow \mathbb{R}$, such that

$$
\mathfrak{E}\left(W_{\sigma, \kappa}(s)\right)(\varrho) \leq \varkappa W_{\sigma, \kappa}(\varrho)
$$

and

$$
\left\|\left(s^{\sigma} I+\vartheta e^{-s \mu}\right)\right\| \leq 1 .
$$

If for each $\varrho \in \mathfrak{B}, \bar{m} \in C\left(\mathfrak{B}, \mathbb{R}^{n}\right)$ is a differentiable function satisfying

$\left\|D_{0}^{\sigma} \overline{\bar{m}}(\varrho)+9 \bar{m}(\varrho-\mu)-g(\varrho)-\mathfrak{E}^{-1}(\bar{m}(s))(\varrho)\right\| \leq \beth W_{\sigma, \kappa}(\varrho), \quad \varrho \in \mathfrak{B}$,

then there is a unique solution $m \in C\left(\mathfrak{B}, \mathbb{R}^{n}\right)$ $Z m \in C\left(\mathfrak{B}, \mathbb{R}^{n}\right)$ to equation (1), such that

$$
\|\bar{m}(\varrho)-m(\varrho)\| \leq \neg \beth W_{\sigma, \kappa}(\varrho),
$$

where $7=x / 1-x$. Then, equation (1) has Hyers-UlamRassais-Wright stability property.

Proof. Define the set $\mathfrak{B}$ as follows:

$$
\mathfrak{S}=\left\{m: \mathfrak{B} \longrightarrow \mathbb{R}^{n}, \quad \text { mis continuous }\right\},
$$

and consider the mapping $d: \mathfrak{E} \times \mathfrak{E} \longrightarrow[0, \infty]$, which is defined as follows:

$d(m, \bar{m})=\operatorname{in}\left\{A \in[0, \infty]:\|m(\varrho)-\bar{m}(\varrho)\| \leq A W_{\sigma, \kappa}(\varrho), \varrho \in \mathfrak{B}\right\}$.

Step 1. We show that $(\mathfrak{E}, d)$ is $[0, \infty]$-valued complete metric space. If for functions $m, \bar{m} \in \mathbb{C}$, we have $d(m, \bar{m})>d(m, v)+d(v, \bar{m})$, then there exists $\varrho_{0} \in \mathfrak{B} \quad$ such that $\left\|m\left(\varrho_{0}\right)-\bar{m}\left(\varrho_{0}\right)\right\|>$ $d(m, v)+d(v, \bar{m}) W_{\sigma, \kappa}\left(\varrho_{0}\right)$. Then, according to the definition $d$, we have

$$
\left\|m\left(\varrho_{0}\right)-\bar{m}\left(\varrho_{0}\right)\right\|>\left\|m\left(\varrho_{0}\right)-v\left(\varrho_{0}\right)\right\|+\left\|v\left(\varrho_{0}\right)-\bar{m}\left(\varrho_{0}\right)\right\|,
$$

which is a contradiction.

Now, we prove that $(\mathfrak{S}, d)$ is a $[0, \infty]$-valued complete. Let $\left\{m_{n}\right\}$ be a Cauchy sequence in $(\mathfrak{S}, d)$. Using $d, \forall \beth>0, \exists N_{\beth} \in \mathbb{N}, \forall m, n \geq N_{\varepsilon}$, and $\forall \varrho \in \mathfrak{B}$, we have

$$
\left\|m_{n}(\varrho)-m_{m}(\varrho)\right\|<\sqsupset W_{\sigma, \kappa}(\varrho) .
$$

Since the function $W_{\sigma, \kappa}$ is continuous to the compact set $\mathfrak{B}$, due to the inequality equation (26), the sequence $\left\{m_{n}\right\}$ uniformly converges to a continuous function of $\mathfrak{C}$ such as $m$. Then, again, using the definition of $d$, the following relation is established for us, wherein for $\beth>0, \exists N_{\beth} \in \mathbb{N}, n \geq N_{\beth}, \varrho \in \mathfrak{C}$,

$$
\left\|m_{n}(\varrho)-m(\varrho)\right\|<\sqsupset W_{\sigma, \kappa}(\varrho) .
$$

It should be noted that we have assumed here that $m$ tends to infinity. As a result, $d\left(m_{n}, m\right) \leq \beth$, and that means $(\mathfrak{c}, d)$ is $[0, \infty]$-valued complete and the first step is over.

Now, according to the main equation, we define the mapping $\widetilde{O}: \mathfrak{E} \longrightarrow \mathfrak{C}$, as follows:

$$
\begin{aligned}
\widetilde{F}(m(\varrho))= & £\left(D_{0}^{\sigma} m(\varrho)+\vartheta m(\varrho-\mu)-g(\varrho)\right)(s) \\
= & \left(s^{\sigma} I+\vartheta e^{-s \mu}\right) \mathfrak{E}(m(\varrho))(s)-s^{\sigma-1} \psi(0)-s^{\sigma-2} \psi^{\prime}(0) \\
& +\vartheta e^{-s \mu} \int_{-\mu}^{0} e^{-s \mu} \psi(\varrho) \mathrm{d} \varrho-\mathfrak{E}(g(\varrho))(s) .
\end{aligned}
$$

Step 2 . We show that $\delta$ is a contractional mapping. If for the functions $m, \bar{m} \in \mathbb{C}$ and the coefficient $A_{m \bar{m}} \in[0, \infty]$, we assume that $d(m, \bar{m}) \leq A_{m \bar{m}}$, the following inequality for $\varrho \in \mathfrak{B}$ is established for us:

$$
\|m(\varrho)-\bar{m}(\varrho)\|<A_{m \bar{m}} W_{\sigma, \kappa}(\varrho),
$$

and using (19) and (20), we have

$$
\begin{aligned}
& \|\delta m(\varrho)-\sigma \bar{m}(\varrho)\|=\|\left(s^{\sigma} I+\vartheta e^{-s \mu}\right) \mathfrak{E}(m(\varrho))(s)-s^{\sigma-1} \psi(0) \\
& -s^{\sigma-2} \psi^{\prime}(0)+\vartheta e^{-s \mu} \int_{-\mu}^{0} e^{-s \mu} \psi(\varrho) \mathrm{d} \varrho-\mathfrak{E}(g(\varrho))(s) \\
& -\left(s^{\sigma} I+\vartheta e^{-s \mu}\right) \mathfrak{E}(\bar{m}(\varrho))(s)+s^{\sigma-1} \psi(0) \\
& +s^{\sigma-2} \psi^{\prime}(0)_{\vartheta} e^{-s \mu} \int_{-\mu}^{0} e^{-s \mu} \psi(\varrho) \mathrm{d} \varrho+\mathfrak{E}(g(\varrho))(s) \\
& =\left\|\left(s^{\sigma} I+\vartheta e^{-s \mu}\right) \mathfrak{E}(m(\varrho))(s)-\left(s^{\sigma} I+\vartheta e^{-s \mu}\right) \mathfrak{E}(\bar{m}(\varrho))(s)\right\| \\
& =\left\|\left(s^{\sigma} I+\vartheta e^{-s \mu}\right) \mathfrak{E}(m(\varrho)-\bar{m}(\varrho))(s)\right\|
\end{aligned}
$$




$$
\begin{aligned}
& \leq\left\|\left(s^{\sigma} I+\vartheta e^{-s \mu}\right) \mathfrak{E}\right\|(m(\varrho)-\bar{m}(\varrho))\|(s)\| \\
& \leq\left\|\left(s^{\sigma} I+\vartheta e^{-s \mu}\right) \mathfrak{E}\left(A_{m \bar{m}} W_{\sigma, \kappa}(s)\right)(\varrho)\right\| \\
& \leq A_{m \bar{m}}\left\|\left(s^{\sigma} I+\vartheta e^{-s \mu}\right) \mathfrak{E}\left(W_{\sigma, \kappa}(s)\right)(\varrho)\right\| \\
& \leq A_{m \bar{m}}\left\|\left(s^{\sigma} I+\vartheta e^{-s \mu}\right) x W_{\sigma, \kappa}(\varrho)\right\| \\
& =A_{m \bar{m}} \chi W_{\sigma, \kappa}(\varrho) .
\end{aligned}
$$

Thus, we have

$$
d(\widetilde{\sigma m}, \widetilde{\sigma m}) \leq \boldsymbol{x} d(m, \bar{m}) .
$$

Since, $0<x<1$, therefore $\sigma$ is a contractional mapping.

Step 3. We will show that $d(\tilde{\delta}, \bar{m})<\infty$. From inequality (21), we have

$$
\begin{aligned}
\|\delta(\bar{m}(\varrho))-\bar{m}(\varrho)\|= & \| \mathfrak{E}\left(D_{0}^{\sigma} \bar{m}(\varrho)+9 \bar{m}(\varrho-\mu)-g(\varrho)-\mathfrak{E}^{-1}(\bar{m}(s))(\varrho) \|\right. \\
= & \|\left(s^{\sigma} I+\vartheta e^{-s \mu}\right) \mathfrak{E}(\bar{m}(\varrho))(s)-s^{\sigma-1} \psi(0) \\
& -s^{\sigma-2} \psi^{\prime}(0)+\vartheta e^{-s \mu} \int_{-\mu}^{0} e^{-s \mu} \psi(\varrho) \mathrm{d} \varrho-\mathfrak{E}(g(\varrho))(s)-\bar{m}(\varrho) \| \\
\leq & \beth \mathfrak{E}\left(W_{\sigma, \kappa}(s)\right)(\varrho) \\
\leq & \beth x W_{\sigma, \kappa}(\varrho) .
\end{aligned}
$$

Consequently,

$$
d(\widetilde{m}, \bar{m}) \leq \beth x<\infty, \quad x<1 .
$$

Therefore, all the conditions of Theorem 6 are true. Then, we have

(1) The sequence $\left\{\delta^{e} m\right\}$ converges to a fixed point such as $m$.

(2) The unique element $m$ is in the set $\mathfrak{V}^{*}=\{\bar{m} \in \mathfrak{V}: d(\widetilde{\varpi}, \bar{m})<\infty\}$ and is the unique fixed point; it means $\delta m=m$ or equivalently

$$
\begin{aligned}
m(\varrho)= & \left(s^{\sigma} I+\vartheta e^{-s \mu}\right) \mathfrak{E}(m(\varrho))(s)-s^{\sigma-1} \psi(0), \\
& -s^{\sigma-2} \psi^{\prime}(0)+\vartheta e^{-s \mu} \int_{-\mu}^{0} e^{-s \mu} \psi(\varrho) \mathrm{d} \varrho-\mathfrak{E}(g(\varrho))(s) .
\end{aligned}
$$

Since $m$ is a continuous function and according to Theorem 5, we have

$$
D_{0}^{\sigma} m(\varrho)=-9 m(\varrho-\mu)+g(\varrho)+\mathfrak{E}^{-1} m(\varrho)(s), \quad \varrho \in \mathfrak{B}:=[0, B] .
$$

(3) According to the inequality (33), we get

$$
d(\bar{m}, m) \leq \frac{1}{1-\varkappa} d(\delta \bar{m}, \bar{m}) \leq \beth \frac{\varkappa}{1-\varkappa} W_{\sigma, \kappa}(\varrho) .
$$

Thus, equation (1) has Hyers-Ulam-Rassais-Wright stability property.

Now, we show the uniqueness of the obtained point. For convenience, we consider

$$
d=\frac{コ x}{1-x},
$$

and let $t$ be another differentiable function satisfying equation (26) and this means that the following equation holds:

$$
D_{0}^{\sigma} \mathfrak{t}(\varrho)=-9 \mathfrak{t}(\varrho-\mu)+g(\varrho)+\mathfrak{E}^{-1}(\mathfrak{t}(s))(\varrho), \quad \varrho \in \mathfrak{B}:=[0, B] .
$$

Now, we show that $\mathbf{t}$ is a fixed point of $\sigma$ and $\mathbf{t} \in \mathfrak{C}^{*}$. Using equation (39), we have $\sigma \mathfrak{t}=\mathfrak{t}$. In the sequel, we show that $d(\widetilde{\delta}, \mathrm{t})<\infty$. For $\bar{m} \in \mathfrak{C}, d(\bar{m}, \mathrm{t})<d$. Using equation (39), we have 


$$
\begin{aligned}
\|\sigma(\bar{m}(\varrho))-\mathfrak{t}(\varrho)\|= & \left\|\mathfrak{E}\left(D_{0}^{\sigma} \bar{m}(\varrho)+9 \bar{m}(\varrho-\mu)-g(\varrho)\right)(s)-\mathfrak{t}(\varrho)\right\| \\
= & \|\left(s^{\sigma} I+\vartheta e^{-s \mu}\right) \mathfrak{E}(\bar{m}(\varrho))(s)-s^{\sigma-1} \psi(0) \\
& -s^{\sigma-2} \psi^{\prime}(0)+\vartheta e^{-s \mu} \int_{-\mu}^{0} e^{-s \mu} \psi(\varrho) \mathrm{d} \varrho-\mathfrak{E}(g(\varrho))(s)-\left(s^{\sigma} I+\vartheta e^{-s \mu}\right) \mathfrak{E}(\mathfrak{t}(\varrho))(s)+s^{\sigma-1} \psi(0) \\
& +s^{\sigma-2} \psi^{\prime}(0)_{\vartheta} e^{-s \mu} \int_{-\mu}^{0} e^{-s \mu} \psi(\varrho) \mathrm{d} \varrho+\mathfrak{E}(g(\varrho))(s) \| \\
= & \left\|\left(s^{\sigma} I+\vartheta e^{-s \mu}\right) \mathfrak{E}(\bar{m}(\varrho))(s)-\left(s^{\sigma} I+\vartheta e^{-s \mu}\right) \mathfrak{E}(\mathfrak{t}(\varrho))(s)\right\| \\
= & \left\|\left(s^{\sigma} I+\vartheta e^{-s \mu}\right) \mathfrak{E}(\bar{m}(\varrho) t-n t q(\varrho))(s)\right\| \\
\leq & \left\|\left(s^{\sigma} I+\vartheta e^{-s \mu}\right) \mathfrak{E}(\|\bar{m}(\varrho)-\mathfrak{t}(\varrho)\|)(s)\right\| \\
\leq & \|\left(s^{\sigma} I+\vartheta e^{-s \mu}\right) \mathfrak{E}\left(d\left(W_{\sigma, \kappa}(s)\right)(\varrho) \|\right. \\
\leq & d\left\|\left(s^{\sigma} I+\vartheta e^{-s \mu}\right) x W_{\sigma, \kappa}(\varrho)\right\| \\
\leq & d x W_{\sigma, \kappa}(\varrho)
\end{aligned}
$$

which means

$$
d(\widetilde{m}, \mathfrak{t}) \leq \varkappa d<\infty .
$$

Example 1. Consider the following nonhomogeneous fractional delay oscillation equation:

$$
\begin{gathered}
D_{0}^{1 / 2} m(\varrho)=-9 m\left(\varrho-\frac{1}{7}\right)+\frac{(\varrho-1)^{-1 / 4} \sin (\varrho-1)}{64(1+\sqrt{\varrho-1})(1+|\varrho|)}+\mathfrak{£}^{-1}(m(s))(\varrho), \\
m(\varrho)=\left[m_{1}(\varrho), m_{2}(\varrho)\right]^{T} \quad \psi(\varrho)=\left[3 \varrho, 4 \varrho^{2}\right]^{T}, \quad-\mu \leq \varrho \leq 0,
\end{gathered}
$$

where $x=1 / 4, \sigma=1 / 2, \mu=1 / 7, B=3 / 5$ and $\vartheta=\left[\begin{array}{cc}3 / 5 & 0 \\ 0 & 2 / 5\end{array}\right]$.

$$
\mathfrak{E}\left(W_{1 / 2, \kappa}(s)\right)(\varrho) \leq \frac{1}{4} W_{1 / 2, \kappa}(\varrho) .
$$

Assume that the following condition is true for the given continuous function:

For $\varrho \in \mathfrak{B}$, let $\bar{m} \in C\left(\mathfrak{B}, \mathbb{R}^{n}\right)$ be a continuous function satisfying

$$
\left\|D_{0}^{1 / 2} \bar{m}(\varrho)+9 \bar{m}\left(\varrho-\frac{1}{7}\right)-\frac{(\varrho-1)^{-1 / 4} \sin (\varrho-1)}{64(1+\sqrt{\varrho-1})(1+|\varrho|)}-\mathfrak{E}^{-1}(m(s))(\varrho)\right\| \leq \beth W_{1 / 2, \kappa}(\varrho), \quad \varrho \in \mathfrak{B},
$$

therefore, according to the definitions given, $\bar{m}$ holds in the following inequality:

$$
\begin{aligned}
& \| \bar{m}(\varrho)-\left(s^{\sigma} I+\vartheta e^{-s \mu}\right) \mathfrak{E}(\bar{m}(\varrho))(s)-s^{\sigma-1} \psi(0) \\
& -s^{\sigma-2} \psi^{\prime}(0)+9 e^{-s \mu} \int_{-\mu}^{0} e^{-s \mu} \psi(\varrho) \mathrm{d} \varrho-\mathfrak{E}\left(\frac{(\varrho-1)^{-1 / 4} \sin (\varrho-1)}{64(1+\sqrt{\varrho-1})(1+|\varrho|)}\right)(s) \| \\
& \leq \beth \mathfrak{E}\left(W_{1 / 2, \kappa}(s)\right)(\varrho) \\
& \leq \frac{1}{4} \sqsupset W_{1 / 2, \kappa}(\varrho),
\end{aligned}
$$


where $\psi(\varrho)=\left[3 \varrho, 4 \varrho^{2}\right]^{T}$. Then, there is a unique solution $m \in C\left(\mathfrak{B}, \mathbb{R}^{n}\right)$ for equation (1), such that
(1) $m$ is the fixed point of the operator $\sigma$, which is defined as follows:

$$
\begin{aligned}
\sigma(m(\varrho))= & \left(s^{\sigma} I+\vartheta e^{-s \mu}\right) \mathfrak{E}(m(\varrho))(s)-s^{\sigma-1} \psi(0) \\
& -s^{\sigma-2} \psi^{\prime}(0)+\vartheta e^{-s \mu} \int_{-\mu}^{0} e^{-s \mu} \psi(\varrho) \mathrm{d} \varrho-\mathcal{E}\left(\frac{(\varrho-1)^{-1 / 4} \sin (\varrho-1)}{64(1+\sqrt{\varrho-1})(1+|\varrho|)}\right)(s) .
\end{aligned}
$$

(2) The fixed point $m$ is unique.

(3) $m$ satisfies in equation (42).

Then,

$$
d(m, \bar{m}) \leq\urcorner \beth,
$$

or

$$
\|\bar{m}(\varrho)-m(\varrho)\| \leq \neg \sqsupset W_{1 / 2, \kappa}(\varrho),
$$

where $7=1 / 3[19-23]$.

\section{Data Availability}

No data were used to support this study.

\section{Conflicts of Interest}

The authors declare that they have no conflicts of interest. The corresponding author is a full-time member of the School of Mathematics, Iran University of Science and Technology, Narmak, Tehran, Iran, and the first author is a Ph.D. student.

\section{Authors' Contributions}

All authors conceived of the study, participated in its design and coordination, drafted the manuscript, participated in the sequence alignment, and read and approved the final manuscript.

\section{References}

[1] K. Diethelm, The Analysis of Fractional Differential Equations. An Application-Oriented Exposition Using Differential operators of Caputo Type, Springer-Verlag, Berlin, Germany, 2010.

[2] D. Yang and C. Bai, "Existence of ground state solutions for an asymptotically 2-linear fractional Schrodinger-Poisson system," Boundary Value Problems, no. 5, p. 9, 2020.

[3] D. Y. Khusainov and G. V. Shuklin, "Relative controllability in systems with pure delay," International Applied Mechanics, vol. 41, no. 2, pp. 210-221, 2005.

[4] D. Y. Khusainov, J. Diblík, M. Růžičková, and J. Lukáčová, "Representation of a solution of the Cauchy problem for an oscillating system with pure delay," Nonlinear Oscillations, vol. 11, no. 2, pp. 276-285, 2008.

[5] M. Li and J. Wang, "Finite time stability and relative controllability of Riemann-Liouville fractional delay differential equations," Mathematical Methods in the Applied Sciences, vol. 42, no. 18, pp. 6607-6623, 2019.
[6] M. Li and J. Wang, "Exploring delayed Mittag-Leffler type matrix functions to study finite time stability of fractional delay differential equations," Applied Mathematics and Computation, vol. 324, pp. 254-265, 2018.

[7] C. Liang, J. Wang, and D. O'Regan, "Representation of a solution for a fractional linear system with pure delay," Applied Mathematics Letters, vol. 77, pp. 72-78, 2018.

[8] V. Kiryakova, "Some special functions related to fractional calculus and fractional (non-integer) order control systems and equations," Facta Universitatis-Series: Automatic Control and Robotics, vol. 7, no. 1, pp. 79-98, 2008.

[9] I. Podlubny, Fractional Differential Equations. An Introduction to Fractional Derivatives, Fractional Differential Equations, to Methods of their Solution and Some of their Applications, Academic Press, San Diego, CA, USA, 1999.

[10] L. Liu, Q. Dong, and G. Li, "Exact solutions and Hyers-Ulam stability for fractional oscillation equations with pure delay," Applied Mathematics Letters, vol. 112, p. 106666, 2021.

[11] J. B. Diaz and B. Margolis, "A fixed point theorem of the alternative, for contractions on a generalized complete metric space," Bulletin of the American Mathematical Society, vol. 74, no. 2, pp. 305-309, 1968.

[12] E. Graily, S. M. Vaezpour, R. Saadati, and Y. J. Cho, "Generalization of fixed point theorems in ordered metric spaces concerning generalized distance," Fixed Point Theory and Applications, vol. 30, p. 8, 2011.

[13] S. Shakeri, L. J. B. Ciric, and R. Saadati, "Common fixed point theorem in partially ordered $L$-fuzzy metric spaces," Fixed Point Theory and Applications, vol. 2010, Article ID 125082, 13 pages, 2010.

[14] L. Ciric, M. Abbas, B. Damjanovic, and R. Saadati, "Common fuzzy fixed point theorems in ordered metric spaces," Mathematical and Computer Modelling, vol. 53, no. 9-10, pp. 1737-1741, 2011.

[15] C. Wang and T.-Z. Xu, "Hyers-Ulam stability of fractional linear differential equations involving Caputo fractional derivatives," Applications of Mathematics, vol. 60, no. 4, pp. 383-393, 2015.

[16] Y. J. Cho and R. Saadati, "Lattictic non-Archimedean random stability of ACQ functional equation," Advances in Difference Equations, vol. 2011, p. 12, 2011.

[17] D. Mihet, R. Saadati, and S. M. Vaezpour, "The stability of an additive functional equation in Menger probabilistic $\phi$-normed spaces," Mathematica Slovaca, vol. 61, no. 5, pp. 817-826, 2011.

[18] Y. J. Cho, C. Park, T. M. Rassias, and R. Saadati, Stability of Functional Equations in Banach Algebras, Springer, Berlin, Germany, 2015. 